\title{
Explicit model predictive control of an active suspension system
}

\author{
J. Theunissen ${ }^{1}$, A. Sorniotti ${ }^{1}$, P. Gruber ${ }^{1}$, S. Fallah ${ }^{1}$, M. Dhaens ${ }^{2}$, K. Reybrouck ${ }^{2}$, C. Lauwerys ${ }^{2}$, \\ B. Vandersmissen ${ }^{2}$, M. Al Sakka ${ }^{2}$ and K. Motte ${ }^{2}$ \\ ${ }^{1}$ University of Surrey, Guildford, UK \\ ${ }^{2}$ Tenneco Automotive Europe bvba, Sint-Truiden, BE
}

\begin{abstract}
Model predictive control (MPC) is increasingly finding its way into industrial applications, due to its superior tracking performance and ability to formally handle system constraints. However, the real-time capability problems related to the conventional implicit model predictive control (i-MPC) framework are well known, especially when targeting low-cost electronic control units (ECUs) for high bandwidth systems, such as automotive active suspensions, which are the topic of this paper. In this context, to overcome the real-time implementation issues of i-MPC, this study proposes explicit model predictive control (e-MPC), which solves the optimization problem off-line, via multi-parametric quadratic programming (mp-QP). e-MPC reduces the on-line algorithm to a function evaluation, which replaces the computationally demanding on-line solution of the quadratic programming $(\mathrm{QP})$ problem. An e-MPC based suspension controller is designed and experimentally validated for a case study Sport Utility Vehicle (SUV), equipped with the active ACOCAR suspension system from the Tenneco Monroe product family. The target is to improve ride comfort in the frequency range of primary ride $(<4 \mathrm{~Hz})$, without affecting the performance at higher frequencies. The proposed e-MPC implementations reduce the root mean square (RMS) value of the sprung mass acceleration by $>40 \%$ compared to the passive vehicle set-up for frequencies $<4 \mathrm{~Hz}$, and by up to $19 \%$ compared to the same vehicle with a skyhook controller on the $0-100 \mathrm{~Hz}$ frequency range.
\end{abstract}

Keywords - Model predictive control, explicit solution, multi-parametric programming, active suspension, ride comfort

\section{1 - Introduction}

Semi-active and active suspensions with hydraulic actuators are widely used on production cars. The permanent challenge of improving ride comfort without increasing hardware costs requires the continuous enhancement of the system intelligence.

The skyhook algorithm is frequently used for primary ride improvement [1]. It is based on the introduction of a virtual damper between the sprung mass and a fixed surface, i.e., the 'sky'. Skyhook can be actuated in full only through an active suspension system, since the vertical velocity of the sprung mass can have a different sign from the relative velocity between the sprung mass and the unsprung mass. The skyhook algorithm was extended for use on controllable dampers, by introducing conditions based on the sign of the ratio of the two speeds [2]. [3] presents an extended skyhook algorithm, in which 
the damper force is a linear combination of a contribution proportional to the vertical velocity of the chassis (skyhook term), and a contribution proportional to the suspension deflection rate.

While skyhook reduces the vehicle body acceleration, the groundhook algorithm improves the unsprung mass dynamics, thus decreasing the oscillations of the vertical tyre load, which are detrimental to the vehicle handling performance [2]. The hybrid skyhook-groundhook controller [4-5] reduces both the dynamic tyre force and body acceleration. [6-7] introduce the semi-active suspension balance logic, targeting a reduction of the sprung mass acceleration. [8] proposes a form of groundhook blended with the balance logic. In the acceleration-driven damping algorithm [9], the shock absorber is deactivated when the body acceleration has opposite sign with respect to the suspension speed.

Model predictive control is a promising option for controllable suspension systems. In particular, i-MPC, in which the optimisation process is run on-line, requires significant computational power within the plant, which makes the practical implementations of i-MPC for high bandwidth systems, including electronic suspensions, difficult. Moreover, the implicit solution cannot be formally analysed a-priori from the viewpoint of its shape, stability and robustness. To the knowledge of the authors, most of the studies proposing i-MPC for electronic suspension systems are limited to simulation-based validations [10-16], with rare exceptions such as [17], using a high-performance $300 \mathrm{MHz}$ Alpha processor.

This paper discusses an active suspension system based on e-MPC (see [18-19] for the theory). With e-MPC the optimisation problem is solved off-line, i.e., explicitly, which reduces the on-line algorithm to a function evaluation. As a consequence, e-MPC requires limited on-line computational power compared to i-MPC, while providing similar control performance and ability to handle constraints. On the other hand, the challenges of e-MPC are the increased design complexity and random-access memory (RAM) demand. e-MPC has already been implemented and, to some extent, experimentally validated on semi-active suspensions [20-23]. In all cases the simple two-mass quarter-car model was used for control system design. However, to the knowledge of the authors, e-MPC has not been proposed for fully active suspensions so far, nor model predictive control for active suspension has ever been implemented on automotive grade microprocessors. This gap is partially covered by this contribution, discussing e-MPC implementations for active suspension systems and their experimental validation on a vehicle demonstrator, including performance comparison with a skyhook controller.

The paper is organised as follows. Section 2 describes the model for control system design. Section 3 deals with the control system and mp-QP problem formulations. Finally, the explicit control law implementation and the experimental results are presented in Section 4.

\section{2 - Model for control system design}

The quarter car model in Fig. 1 is used as a basis for control system design, i.e., as prediction model. The active suspension component is a hydraulic actuator, generating an ideal force input, $u(t)$, without delays or actuation dynamics, where $t$ is time.

The equations of motion are:

$$
\left\{\begin{array}{c}
m_{1} \ddot{x}_{1}+k_{1}\left(x_{1}-x_{2}\right)+c_{1}\left(\dot{x}_{1}-\dot{x}_{2}\right)+u=0 \\
m_{2} \ddot{x}_{2}+k_{1}\left(x_{2}-x_{1}\right)+k_{2}\left(x_{2}-w\right)+c_{1}\left(\dot{x}_{2}-\dot{x}_{1}\right)+c_{2}\left(\dot{x}_{2}-\dot{w}\right)-u=0
\end{array}\right.
$$


where $m_{1}$ and $m_{2}$ are the sprung and unsprung masses; $k_{1}, k_{2}, c_{1}$ and $c_{2}$ are the vertical suspension stiffness, the vertical tyre stiffness, the vertical damping coefficient associated with the passive suspension components, and the vertical tyre damping coefficient; and $x_{1}, x_{2}$ and $w$ are the vertical displacement of the sprung mass, the vertical displacement of the unsprung mass, and the vertical displacement of the road profile.

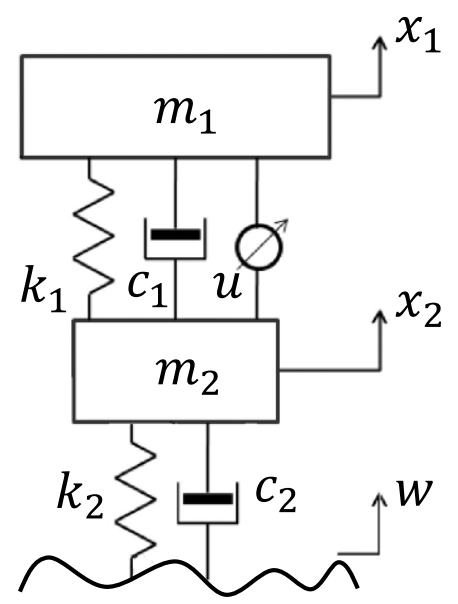

Fig. 1 - Quarter car model with active suspension system.

The system can be converted into a continuous time state-space notation:

$$
\left\{\begin{array}{c}
\dot{\boldsymbol{x}}(t)=\boldsymbol{A} \boldsymbol{x}(t)+\boldsymbol{B} u(t)+\boldsymbol{E} \boldsymbol{W}(t) \\
y(t)=\boldsymbol{C} \boldsymbol{x}(t)+\boldsymbol{D} u(t)
\end{array}\right.
$$

where $\boldsymbol{A}, \boldsymbol{B}, \boldsymbol{C}$ and $\boldsymbol{D}$ are the system matrices, and $\boldsymbol{E}$ is the disturbance matrix. The road input is represented by the column vector $\boldsymbol{W}(t)=\left[\begin{array}{cc}w & \dot{w}\end{array}\right]^{T}$, where $\dot{w}$ is the vertical velocity of the road at the tyre contact point. The output, $y(t)=\ddot{x}_{1}$, is the acceleration of the sprung mass.

e-MPC is based on a state feedback law. Hence, the controller performance depends on the accuracy and appropriate selection of the measured or estimated states. In the specific implementation of this study, the state vector, $\boldsymbol{x}(t)=\left[\begin{array}{llll}x_{1} & \dot{x}_{1} & x_{1}-x_{2} & \dot{x}_{1}-\dot{x}_{2}\end{array}\right]^{T}$, contains the position and speed of the sprung mass, and the suspension displacement and deflection rate. The estimates of $x_{1}$ and $\dot{x}_{1}$ are obtained through the band-pass filtering and mathematical integration of the vertical acceleration measurements of the vehicle body. $x_{1}-x_{2}$ is estimated through the direct measurement of the active suspension actuator displacement, and consideration of the suspension installation ratio. $\dot{x}_{1}-\dot{x}_{2}$ is obtained through differentiation of $x_{1}-x_{2}$ by using the hybrid smooth derivative method [24]. $\boldsymbol{E}$ represents the influence of the unknown disturbances $w$ and $\dot{w}$, and is neglected during the e-MPC design. 


\section{3 - Control system formulation}

\section{1 - System prediction}

The discrete state-space formulation of the vehicle model is:

$$
\left\{\boldsymbol{x}[\mathrm{k}+1]=\boldsymbol{A}_{\boldsymbol{d}} \boldsymbol{x}[\mathrm{k}]+\boldsymbol{B}_{\boldsymbol{d}} u[\mathrm{k}]\right.
$$

Given the initial state, $\boldsymbol{x}[\mathrm{k}]$, the initial control input, $u[\mathrm{k}]$, and the system (3), the output over the prediction horizon, $\widehat{\boldsymbol{y}}$, is calculated as:

$$
\begin{aligned}
\widehat{\boldsymbol{y}}=\left[\begin{array}{c}
\boldsymbol{C}_{\boldsymbol{d}} \boldsymbol{A}_{\boldsymbol{d}} \\
\boldsymbol{C}_{\boldsymbol{d}} \boldsymbol{A}_{\boldsymbol{d}}^{2} \\
\vdots \\
\boldsymbol{C}_{\boldsymbol{d}} \boldsymbol{A}_{\boldsymbol{d}}^{p}
\end{array}\right]_{\mathrm{p} \times 1} \boldsymbol{x}[\mathrm{k}]+\left[\begin{array}{c}
\boldsymbol{C}_{\boldsymbol{d}} \boldsymbol{B}_{\boldsymbol{d}} \\
\boldsymbol{C}_{\boldsymbol{d}} \boldsymbol{A}_{\boldsymbol{d}} \boldsymbol{B}_{\boldsymbol{d}} \\
\vdots \\
\boldsymbol{C}_{\boldsymbol{d}} \boldsymbol{A}_{\boldsymbol{d}}^{p-1} \boldsymbol{B}_{\boldsymbol{d}}
\end{array}\right]_{\mathrm{p} \times 1} u[\mathrm{k}] \\
+\left[\begin{array}{cccc}
\boldsymbol{D}_{\boldsymbol{d}} & 0 & 0 & 0 \\
\boldsymbol{C}_{\boldsymbol{d}} \boldsymbol{B}_{\boldsymbol{d}} & \ddots & 0 & 0 \\
\vdots & \ddots & \ddots & 0 \\
\boldsymbol{C}_{\boldsymbol{d}} \boldsymbol{A}_{d}{ }^{p-2} \boldsymbol{B}_{\boldsymbol{d}} & \cdots & \boldsymbol{C}_{\boldsymbol{d}} \boldsymbol{B}_{\boldsymbol{d}} & \boldsymbol{D}_{\boldsymbol{d}}
\end{array}\right]_{\mathrm{p} \times \mathrm{n}} \widehat{\boldsymbol{u}}
\end{aligned}
$$

which, more concisely, is expressed as:

$$
\widehat{\boldsymbol{y}}=\boldsymbol{\Lambda} \boldsymbol{x}[\mathrm{k}]+\boldsymbol{\Theta}_{\boldsymbol{u} \boldsymbol{0}} u[\mathrm{k}]+\boldsymbol{\Theta}_{\boldsymbol{u}} \widehat{\boldsymbol{u}}
$$

where:

$$
\widehat{\boldsymbol{y}}=\left[\begin{array}{c}
y[\mathrm{k}+1] \\
\vdots \\
y[\mathrm{k}+\mathrm{p}]
\end{array}\right], \quad \widehat{\boldsymbol{u}}=\left[\begin{array}{c}
u[\mathrm{k}+1] \\
\vdots \\
u[\mathrm{k}+\mathrm{n}]
\end{array}\right]
$$

$\mathrm{n}$ and $\mathrm{p}$ are the control horizon and prediction horizon, respectively. The states over the prediction horizon, $\widehat{\boldsymbol{x}}$, are given by:

$$
\widehat{\boldsymbol{x}}=\boldsymbol{\Psi} \boldsymbol{x}[\mathrm{k}]+\boldsymbol{\Omega}_{\boldsymbol{u} \boldsymbol{0}} u[\mathrm{k}]+\boldsymbol{\Omega}_{\boldsymbol{u}} \widehat{\boldsymbol{u}}
$$

in which: 


$$
\widehat{x}=\left[\begin{array}{c}
x[\mathrm{k}+1] \\
\vdots \\
x[\mathrm{k}+\mathrm{p}]
\end{array}\right]
$$

$\widehat{\boldsymbol{u}}$ is the input over the prediction horizon. The matrices $\boldsymbol{\Psi}, \boldsymbol{\Omega}_{\boldsymbol{u} \mathbf{0}}$ and $\boldsymbol{\Omega}_{\mathbf{u}}$ are calculated from the system model (3).

\section{2 - Objective function}

The general goal of suspension design is the optimisation of ride comfort, suspension rattle space and road holding. The ride comfort improvement is achieved through the reduction of the vehicle body acceleration levels, while limiting chassis motion as much as possible. Hence, this study uses a cost function penalising $\ddot{x}_{1}, x_{1}, x_{1}-x_{2}$ and the control effort $u$. The continuous form of the performance index to be minimised, $J_{e-M P C}$, is:

$$
J_{e-M P C}=\int_{0}^{T}\left(\frac{\rho_{1}}{\mathrm{n}_{1}} \ddot{x}_{1}^{2}+\frac{\rho_{2}}{\mathrm{n}_{2}}\left(x_{1}-x_{2}\right)^{2}+\frac{\rho_{3}}{\mathrm{n}_{3}} x_{1}^{2}+\frac{\rho_{4}}{\mathrm{n}_{4}} u^{2}\right) d t
$$

where $\rho_{i}$ and $n_{i}$ are the weighting and normalisation factors, respectively, and $T$ is the period of observation, i.e., the duration of the prediction horizon.

\section{3 - mp-QP problem formulation}

$J_{e-M P C}$ is re-arranged to be consistent with the discretised system prediction formulation of Section 3.1. $J_{e-M P C}$ is quadratic, and is used for the following minimisation problem:

$$
\min _{\widehat{\boldsymbol{u}}}\left(\widehat{\boldsymbol{y}}^{T} \mathbf{Q}_{\mathbf{1}} \widehat{\boldsymbol{y}}+\widehat{\boldsymbol{x}}^{\mathbf{T}} \mathbf{Q}_{\mathbf{2}} \widehat{\boldsymbol{x}}+\widehat{\boldsymbol{u}}^{T} \mathbf{R} \widehat{\boldsymbol{u}}\right)
$$

where $\mathbf{Q}_{\mathbf{1}}, \mathbf{Q}_{\mathbf{2}}$ and $\mathbf{R}$ contain the factors $\rho_{\mathrm{i}}$ and $\mathrm{n}_{\mathrm{i}}$ of (9). Through appropriate re-arrangements and simplifications, the model predictive control formulation can be represented by the following QP problem:

$$
\min _{\widehat{\boldsymbol{u}}} \frac{1}{2} \widehat{\boldsymbol{u}}^{T} \mathbf{H} \widehat{\boldsymbol{u}}+\boldsymbol{x}^{\prime}[\mathrm{k}]^{T} \mathbf{F} \widehat{\boldsymbol{u}}
$$

where $\mathbf{H}$ is the Hessian matrix, and $\mathbf{F}$ includes the physical system parameters and the weighting and normalisation factors. $\boldsymbol{x}^{\prime}[\mathrm{k}]$ contains the initial states of the system as well as the initial actuator force.

A conventional i-MPC would execute an on-line optimisation at each time step, $\Delta t$, for a given value of $\boldsymbol{x}^{\prime}[\mathrm{k}]$, and the control law $u=u\left(\boldsymbol{x}^{\prime}\right)$ would be implicitly obtained by the QP solver. In the e-MPC case the optimisation is performed off-line. The QP is solved for the defined range of $\boldsymbol{x}^{\prime}$, which generates the explicit solution, $u=u\left(\boldsymbol{x}^{\prime}\right)$. The optimisation problem becomes an mp-QP problem, generally described as follows: 


$$
\min _{\widehat{\boldsymbol{u}}} \frac{1}{2} \widehat{\boldsymbol{u}}^{T} \mathbf{H} \widehat{\boldsymbol{u}}+\boldsymbol{x}^{\prime T} \mathbf{F} \widehat{\boldsymbol{u}}+\frac{1}{2} \boldsymbol{x}^{\prime T} \mathbf{Y} \boldsymbol{x}^{\prime}
$$

subject to:

$$
\mathrm{N} \widehat{u} \leq \mathrm{M}_{1}+\mathrm{M}_{2} \boldsymbol{x}^{\prime}
$$

where $\mathbf{Y}, \mathbf{N}, \mathbf{M}_{\mathbf{1}}$ and $\mathbf{M}_{\mathbf{2}}$ are constant matrices. The constraints are typically related to actuator force and its rate. The last term in (12) is neglected, since it does not depend on $\widehat{\boldsymbol{u}}$.

The solutions of the mp-QP problem is the piecewise affine function $U^{*}$, which associates the corresponding $\widehat{\boldsymbol{u}}$ to each $\boldsymbol{x}^{\prime}$. e-MPC uses the control input at the first time step only, i.e., $u\left(\boldsymbol{x}^{\prime}\right)=$ [I $\quad 0 \cdots 0] U^{*}$. Hence, the explicit representation of the control action is a piecewise affine state feedback law defined on a polyhedral partition of the state-space:

$$
u\left(x^{\prime}\right)= \begin{cases}\mathbf{L}_{\mathbf{1}} \boldsymbol{x}^{\prime}+\mathbf{p}_{\mathbf{1}}, & \mathbf{0}_{\mathbf{1}} \boldsymbol{x}^{\prime} \leq \mathbf{S}_{\mathbf{1}} \\ \vdots & \vdots \\ \mathbf{L}_{\mathbf{j}} \boldsymbol{x}^{\prime}+\mathbf{p}_{\mathbf{k}}, & \mathbf{0}_{\mathbf{M}} \boldsymbol{x}^{\prime} \leq \mathbf{S}_{\mathbf{j}}\end{cases}
$$

where $\mathbf{L}_{\mathbf{i}}, \mathbf{p}_{\mathbf{i}}, \mathbf{O}_{\mathbf{i}}$ and $\mathbf{S}_{\mathbf{i}}$ are constant matrices.

\section{4 - Distributed controller}

To reduce the off-line computation time and the on-line RAM requirements of the explicit solution, four controllers based on quarter car models are used, i.e., one controller for each suspension system. A prediction model including pitch dynamics would imply a larger number of parameters per mp-QP problem, and more demanding RAM requirements.

\section{4 - Controller implementation and experimental evaluation}

\section{1 - Vehicle demonstrator}

The developed controller was implemented and experimentally validated on a sport utility vehicle (SUV) demonstrator (see Fig. 2) with a hydraulic active suspension system, i.e., the Tenneco Monroe intelligent suspension - ACOCAR. The sensor set and valves are identical to those of the Tenneco CVSA2 (continuously variable semi-active system with two valves) suspension technology [25]. The ACOCAR actuators are pressurised by means of a pump, which allows inputting energy into the system and actively controlling the actuation forces. The low level actuator controller, which calculates the valve currents and reference pump speed as functions of the actuator force demand and speed, already exists and is fully calibrated.

The SUV demonstrator was used to compare the experimental performance of the ACOCAR e-MPC implementations with those of: 
- The passive set-up of the car. This was obtained by applying zero currents to the actuation valves, which represents the fail-safe state of the ACOCAR system, corresponding to a suspension tuning that is very close to the one of the passive version of the SUV.

- A conventional production-ready skyhook algorithm for active suspensions, configured with high gains. The adopted skyhook damping coefficients for the heave, pitch and roll motions are respectively $10,000 \mathrm{Ns} / \mathrm{m}, 12,000 \mathrm{Nms} / \mathrm{rad}$ and $12,000 \mathrm{Nms} / \mathrm{rad}$.

The comparison was carried out for the excitation profile of a typical ride comfort road, i.e., the Blauwe Kei road at the Ford Lommel proving ground in Belgium, which was reproduced by means of a Schenck Instron 4-poster test rig, exciting the SUV demonstrator.

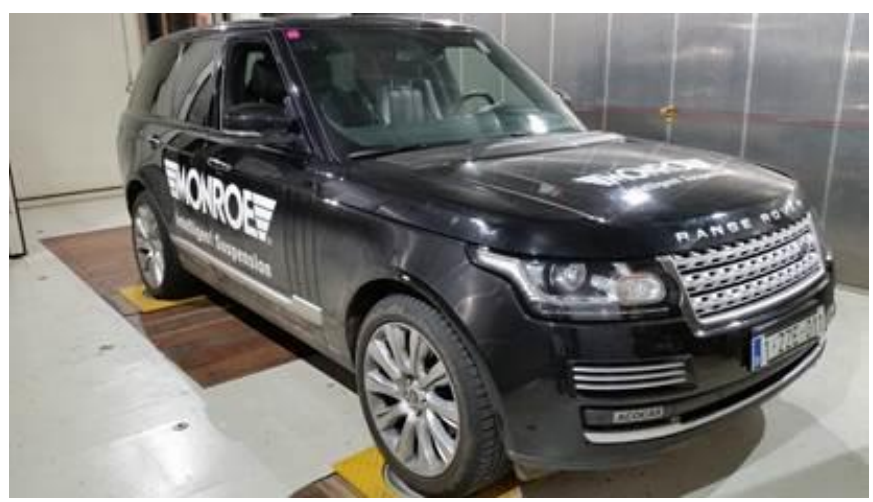

Fig. 2 - The SUV demonstrator on the 4-poster test rig.

\section{2 - Controller implementation}

The first step of the e-MPC implementation process was the validation of the front and rear quarter car models. This is based on experimental results obtained from measurements on the 4-poster test rig, for a set-up of the SUV demonstrator with the least possible damping.

The following variables are available in the SUV demonstrator:

- Heave, pitch and roll displacements at the centre of gravity of the vehicle body, calculated from the vertical acceleration measurements at three points of the sprung mass. Coordinate transformations are applied to obtain the velocities and displacements of the top suspension mounts.

- Suspension displacements, calculated from the suspension rotation sensors and calibration maps.

A good modelling match was achieved up to $\sim 15 \mathrm{~Hz}$, which is in line with the prediction model bandwidth. In particular, the dynamics of the vehicle body and unsprung masses at the resonance frequencies of $\sim 1-1.5 \mathrm{~Hz}$ and $\sim 10-12 \mathrm{~Hz}$, respectively, are captured well.

Then the mp-QP problems for the front and rear suspensions were solved with the multi-parametric toolbox 3 (MPT3) [26], for different sets of coefficients of $J_{e-M P C}$. Simulations of the implemented controllers with an experimentally validated vehicle model for control system assessment were used along the Blauwe Kei profile for the identification of the coefficients of $J_{e-M P C}$ providing the most desirable e-MPC behaviour. The performance assessment was carried out with the same RMS-based performance indicators that will be reported in the following Table I (see Section 4.4). 
In the control design phase a prediction horizon $p=5$ and a control horizon $n=5$ were adopted, with a controller sample time $\Delta t=10 \mathrm{~ms}$. Each actuator force was constrained to $\pm 5,000 \mathrm{~N}$. The suspension installation ratio is applied to calculate the actuator reference force, from the force demand at the wheel output by the controller, i.e., $u(t)$. In particular, at the completion of the process, two e-MPC settings, called e-MPC ${ }_{1}$ and e-MPC 2 with objective function tunings $J_{e-M P C_{1}}$ and $J_{e-M P C_{2}}$, were considered for further experimental evaluation:

- $\quad$ e-MPC ${ }_{1}$. Compared to the skyhook, this setting reduces $\ddot{x}_{1}$ for frequencies $<4 \mathrm{~Hz}$, without excessively increasing the acceleration levels above that frequency. The latter specification is based on the experience of Tenneco, showing that active suspensions with hydraulic actuators in parallel to the springs, as it is the case here, tend to reduce ride comfort at medium-high frequencies. This is caused by the typically limited actuator bandwidth $(\sim 8 \mathrm{~Hz})$, the significant non-linearities, and the fact that the actuator force is delivered by changing the damping coefficient.

- $\quad$ e-MPC 2 . With respect to the skyhook, this setting targets similar performance around the resonance frequency of the sprung mass $(1-1.5 \mathrm{~Hz})$, and the reduction of the acceleration levels at frequencies $>4 \mathrm{~Hz}$. In comparison with the e-MPC 1 , the e-MPC $\mathrm{M}_{2}$ increases the penalty on $\ddot{x}_{1}$ and reduces the penalty on $x_{1}$ in $J_{e-M P C}$.

\section{3 - The explicit solution of the e-MPC$C_{1}$}

This section discusses the explicit solution of the e-MPC ${ }_{1}$. The control law, $u=u\left(\boldsymbol{x}^{\prime}\right)$, consists of a set of functions with affine gains over 1217 polyhedral regions within the state-space. Fig. 3 shows the statespace partition, sliced at $x_{1}-x_{2}=0$ and $\dot{x}_{1}-\dot{x}_{2}=0$. In region 1 the control law varies as a function of $x_{1}$ and $\dot{x}_{1}$. In regions $2,3,4$ and 5 the actuator force is saturated.

Fig. 3 also reports the simulation results for the front left SUV corner in the passive and e-MPC $\mathrm{C}_{1}$ set-ups, in the form of state trajectories for the Blauwe Kei road input. Interestingly, in the e-MPC ${ }_{1}$ case the system operation is limited to one region, i.e., the first sub-partition bounded by:

$\left[\begin{array}{cccc}0 & 1 & 0 & 0 \\ -0.9678 & 0.2422 & 0.0682 & -0.0052 \\ -0.9690 & 0.1225 & 0.2146 & -0.0041 \\ -0.9493 & 0.0835 & 0.3030 & -0.0024 \\ -0.9402 & 0.0673 & 0.3338 & -0.0006 \\ -0.9483 & 0.0607 & 0.3116 & 0.0010 \\ \vdots & \vdots & \vdots & \vdots \\ 0.9989 & 0.0315 & -0.0331 & -0.0029\end{array}\right]_{25 \times 4} \quad \boldsymbol{x}^{\prime} \leq\left[\begin{array}{c}2 \\ 0.5330 \\ 0.2914 \\ 0.2129 \\ 0.1843 \\ 0.1805 \\ \vdots \\ 0.0451\end{array}\right]$




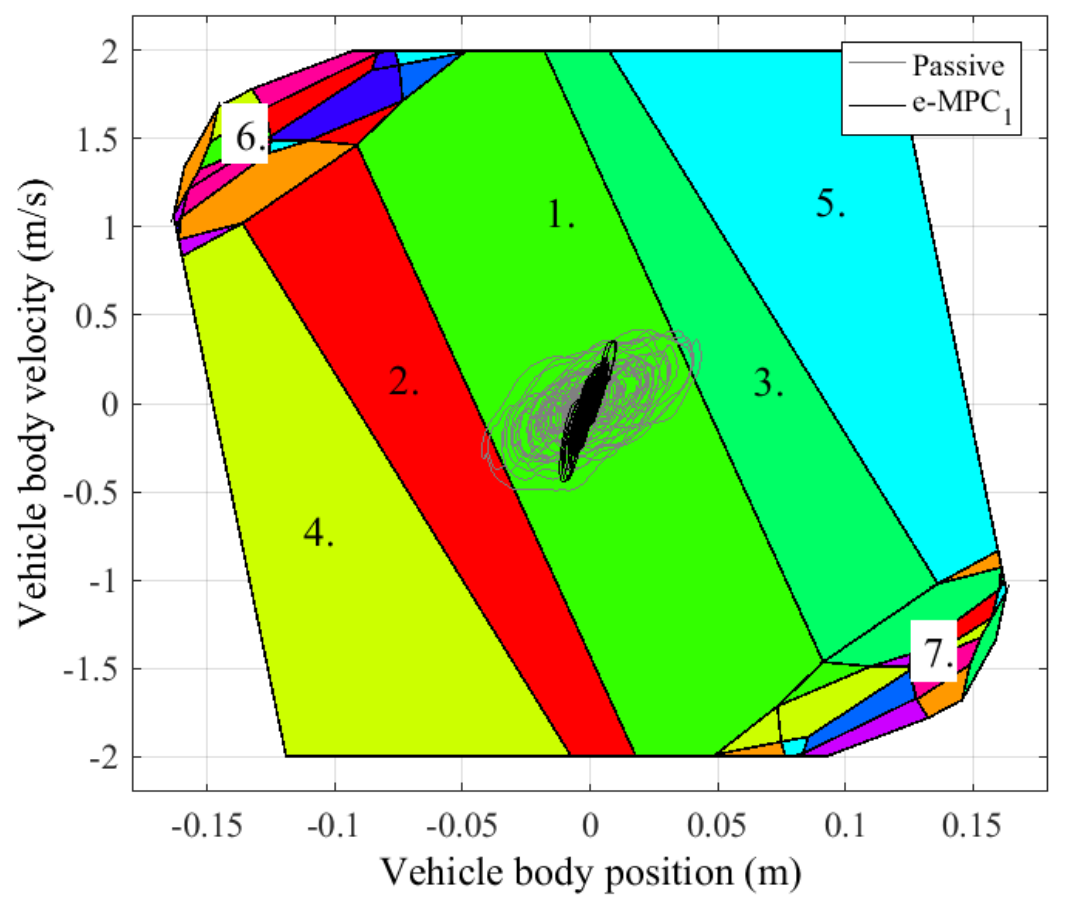

Fig. 3 - The e-MPC $C_{1}$ state-space partition sliced at $x_{1}-x_{2}=0$ and $\dot{x}_{1}-\dot{x}_{2}=0$, with the simulated trajectories of the front left SUV corner with the passive set-up and e-MPC 1 set-up on the Blauwe Kei road.

Although Fig. 3 depicts only a two-dimensional slice of the four-dimensional state-space partition, this behaviour was verified on the four-dimensional partition. As a consequence, on the specific road the control law could be replaced by the following single affine function of the states:

$$
\begin{aligned}
& u\left(\boldsymbol{x}^{\prime}\right)=\left[\begin{array}{llll}
1.1086 \cdot 10^{5} & 0.0350 \cdot 10^{5} & -0.0368 \cdot 10^{5} & -0.0033 \cdot 10^{5}
\end{array}\right] \boldsymbol{x}^{\prime} \\
& +\left[9.0949 \cdot 10^{-13}\right]
\end{aligned}
$$

resulting in a significant reduction of the RAM requirements. Obviously, this would not be advisable during operation on more aggressive road profiles. The potential simplification of the e-MPC control law, either through formal and systematic methods (see [27]) or the empirical observation of the most commonly used sub-partitions, will be the topic of future research.

The whole explicit e-MPC $\mathrm{M}_{1}$ solution from the MPT3 toolbox was uploaded on the dSPACE AutoBox rapid control prototyping unit of the SUV demonstrator.

\section{4 - Experimental results and comparisons}

This section reports the experimental SUV results on the 4-poster test rig along the assessed mission profile. In particular, Fig. 4 and Fig. 5 plot the frequency response characteristics of the power spectral densities (PSDs) of the heave position and heave acceleration of the centre of gravity of the sprung mass for the four considered set-ups, i.e., passive, skyhook, e-MPC 1 and e- $\mathrm{MPC}_{2}$. Table I shows the corresponding root mean square (RMS) values for the frequency ranges below and above $4 \mathrm{~Hz}$, 
corresponding to the so-called primary ride and secondary ride, and for the $0-100 \mathrm{~Hz}$ ride comfort frequency spectrum.

In the $0-4 \mathrm{~Hz}$ frequency range, the e-MPC ${ }_{1}$ reduces the sprung mass heave acceleration by $43 \%$ with respect to the passive set-up, and by $26 \%$ compared to the skyhook, which is a major primary ride enhancement. On the other hand, above $4 \mathrm{~Hz}$ the skyhook strategy increases the vertical acceleration by 79\% compared to the passive set-up. This phenomenon, which is well-known to the technical specialists of Tenneco, is attributed to the limited actuation dynamics of the hydraulic active suspension system. In fact, simulations of the system response with actuators with better dynamic properties did not show such a trend. This behaviour brings a deterioration of the secondary ride. In the same frequency range $(>4$ $\mathrm{Hz}$ ) the e-MPC ${ }_{1}$ shows an increase in the vertical acceleration level of $65 \%$ compared to the passive setup, which is an $8 \%$ reduction of the secondary ride problem of the skyhook. On the $0-100 \mathrm{~Hz}$ frequency range, the e- $\mathrm{MPC}_{1}$ reduces the skyhook vibration levels by $11 \%$.

The e-MPC 2 was implemented with the purpose of attenuating the secondary ride issues of the skyhook and $\mathrm{e}-\mathrm{MPC}_{1}$, while providing good primary ride performance. Fig. 5 shows that at approximately the resonance frequency of the sprung mass, i.e., at $1-1.5 \mathrm{~Hz}$, the e- $\mathrm{MPC}_{2}$ and the skyhook give origin to similar responses. The e-MPC $\mathrm{MP}_{2}$ improves the skyhook acceleration performance by $22 \%$ in the $0-4 \mathrm{~Hz}$ frequency range, and by $19 \%$ above $4 \mathrm{~Hz}$. Moreover, on the whole frequency range the e-MPC $\mathrm{M}_{2}$ produces lower acceleration levels than the e-MPC 1 , which is expected given the increased penalty on $\ddot{x}_{1}$ in $J_{e-M P C_{2}}$. The conclusion is that for the given actuators the e-MPC 2 conjugates a significant enhancement of the primary ride, without an excessive penalisation of the secondary ride performance.

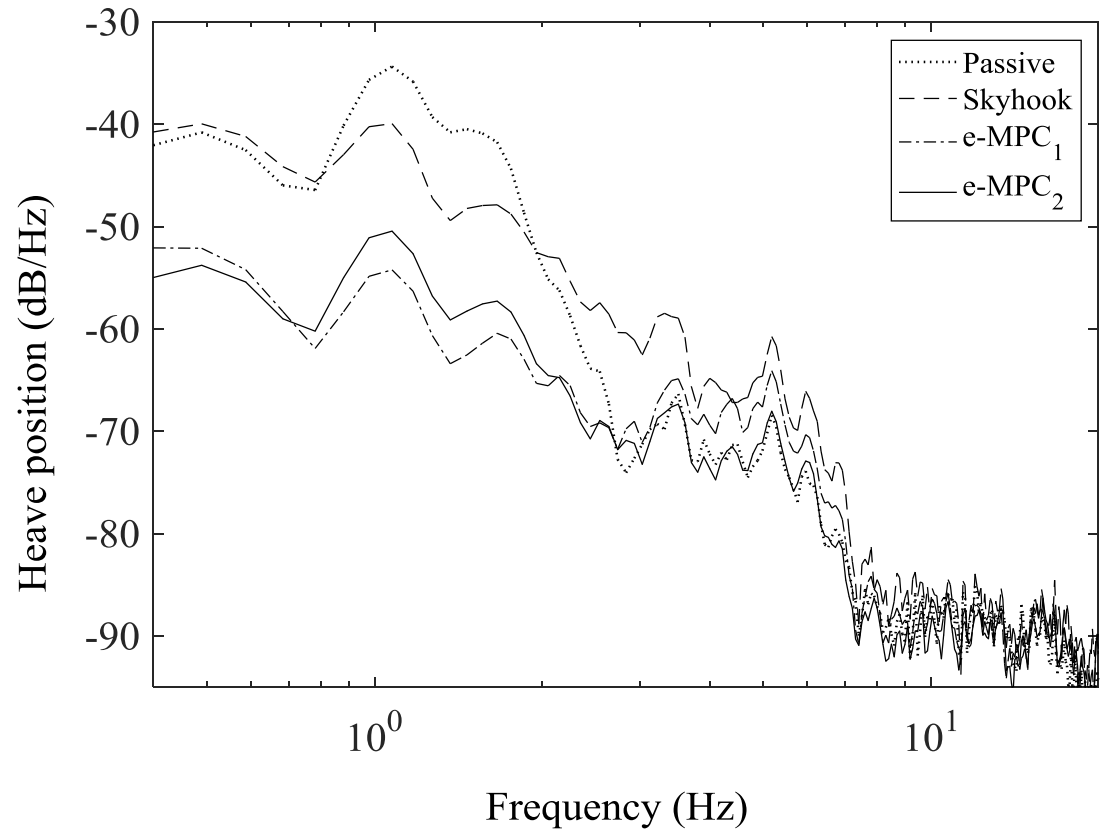

Fig. 4 - PSD of the heave displacement of the centre of gravity of the sprung mass for the passive, skyhook, e-MPC 1 and e-MPC 2 set-ups. 


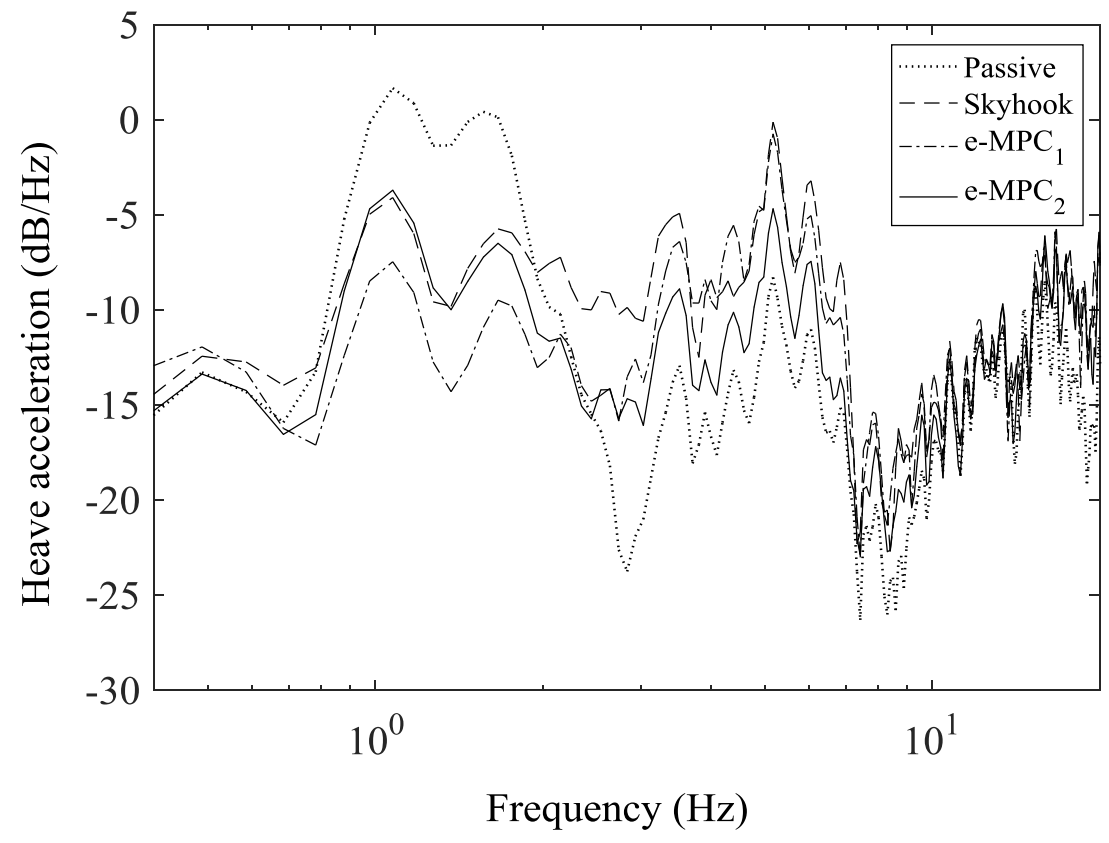

Fig. 5 - PSD of the heave acceleration of the centre of gravity of the sprung mass for the passive, skyhook, e-MPC ${ }_{1}$ and e-MPC 2 set-ups.

Table I - RMS values for the heave position and acceleration of the centre of gravity of the sprung mass for the passive, skyhook, e-MPC 1 and e-MPC 2 set-ups.

\begin{tabular}{|c|c|c|c|c|}
\hline & \multicolumn{4}{|c|}{ RMS values } \\
\hline & Passive & $\begin{array}{l}\text { Skyhook } \\
\text { (wrt Passive) }\end{array}$ & $\begin{array}{c}\text { e-MPC } \\
\text { (wrt Passive/Skyhook) } \\
\end{array}$ & $\begin{array}{c}\mathrm{e}-\mathrm{MPC}_{2} \\
\text { (wrt Passive/Skyhook) }\end{array}$ \\
\hline $\begin{array}{l}\text { Heave position: } 0-4 \mathrm{~Hz} \\
(\mathrm{~m})\end{array}$ & 0.0132 & $\begin{array}{c}0.0099 \\
(-25 \%)\end{array}$ & $\begin{array}{c}0.0024 \\
(-82 \% /-75 \%)\end{array}$ & $\begin{array}{c}0.0023 \\
(-82 \% /-76 \%)\end{array}$ \\
\hline $\begin{array}{l}\text { Heave position: } 4-100 \mathrm{~Hz} \\
(\mathrm{~m})\end{array}$ & 0.0004 & $\begin{array}{l}0.0009 \\
(+125 \%)\end{array}$ & $\begin{array}{c}0.0006 \\
(+50 \% /-33 \%)\end{array}$ & $\begin{array}{c}0.0004 \\
(+0 \% /-55 \%)\end{array}$ \\
\hline $\begin{array}{l}\text { Heave position: } 0-100 \mathrm{~Hz} \\
(\mathrm{~m})\end{array}$ & 0.0132 & $\begin{array}{c}0.0099 \\
(-25 \%)\end{array}$ & $\begin{array}{c}0.0024 \\
(-82 \% /-75 \%)\end{array}$ & $\begin{array}{c}0.0023 \\
(-82 \% /-76 \%)\end{array}$ \\
\hline $\begin{array}{l}\text { Heave acceleration: } 0-4 \mathrm{~Hz} \\
\left(\mathrm{~m} / \mathrm{s}^{2}\right)\end{array}$ & 1.01 & $\begin{array}{c}0.77 \\
(-24 \%)\end{array}$ & $\begin{array}{c}0.57 \\
(-43 \% /-26 \%)\end{array}$ & $\begin{array}{c}0.60 \\
(-41 \% /-22 \%)\end{array}$ \\
\hline $\begin{array}{l}\text { Heave acceleration: } 4-100 \mathrm{~Hz} \\
\qquad\left(\mathrm{~m} / \mathrm{s}^{2}\right)\end{array}$ & 0.91 & $\begin{array}{c}1.63 \\
(+79 \%)\end{array}$ & $\begin{array}{c}1.50 \\
(+65 \% /-8 \%)\end{array}$ & $\begin{array}{c}1.32 \\
(+45 \% /-19 \%)\end{array}$ \\
\hline $\begin{array}{l}\text { Heave acceleration: } 0-100 \mathrm{~Hz} \\
\qquad\left(\mathrm{~m} / \mathrm{s}^{2}\right)\end{array}$ & 1.36 & $\begin{array}{c}1.80 \\
(+32 \%)\end{array}$ & $\begin{array}{c}1.60 \\
(+18 \% /-11 \%)\end{array}$ & $\begin{array}{c}1.45 \\
(+6 \% /-19 \%)\end{array}$ \\
\hline
\end{tabular}




\section{5 - Conclusion}

To the knowledge of the authors, for the first time this paper discussed the application of e-MPC to an active suspension system for passenger cars, mainly targeting primary ride improvements. Multiparametric quadratic programming was used to solve the control problem formulation, based on a quarter car model. The solution is represented by explicit control laws, based on state feedback. e-MPC brings a reduction of the computational requirements of the control system hardware with respect to i-MPC, as the on-line implementation consists of a function evaluation. The results show significant benefits of the developed controllers with respect to a pre-existing skyhook algorithm. In fact, the e-MPC 1 and e-MPC 2 implementations reduce the vehicle body acceleration levels by $26 \%$ and $22 \%$, respectively, in the frequency range below $4 \mathrm{~Hz}$, and by $8 \%$ and $19 \%$, respectively, above $4 \mathrm{~Hz}$. Future developments will focus on the systematic fine-tuning of the objective function for e-MPC design, and the assessment of the controllers on different actuation hardware.

\section{Acknowledgement}

The authors would like to thank Tenneco Automotive Europe (Sint-Truiden, Belgium) for their contribution to this research. This paper is a modified version of the conference paper [28].

\section{References}

1. Karnopp D., Crosby M.J. and Harwood RA. Vibration control using semi-active force generators, J. Eng. Ind., 1974, 96(2), 619-626.

2. Valasek M. and Novak M. Ground Hook for Semi-Active Damping of Truck's Suspension, Proceedings of the CTU Workshop 96, Engineering Mechanics, 1996, 22-24.

3. Lauwerys $\mathrm{C}$. Control of active and semi-active suspension systems for passenger cars, $\mathrm{PhD}$ thesis, Catholic University of Leuven, 2005.

4. Ahmadian M. A hybrid semi-active control for secondary suspension applications, Proceedings of the $6^{\text {th }}$ ASMA Symposium on Advanced Automotive Technologies, 1997, 16-21.

5. Goncalves F.D. and Ahmadian M. A hybrid control policy for semi-active vehicle suspension, J. Shock and Vib., 2003, 10(1), 59-69.

6. Rakheja S. and Sankar S. Vibration and Shock Isolation Performance of a Semi-Active On-Off Damper, J. Vib., Acoust., Stress, Reliab., 1985, 107(4), 398-403.

7. Stammers C.W. and Sireteanu T. Vibration control of machines by using semi-active dry friction damping, J. Snd. and Vib., 1997, 209(4), 671-684.

8. Koo J.H., Setareh M. and Murray T.M. In search of suitable control methods for semi-active tuned vibration absorbers, J. Vib. and Ctrl., 2004, 10, 163-174.

9. Savaresi S.M. and Silani E. On the Optimal Predictive Control Algorithm for Comfort-Oriented Semi-Active Suspensions, SAE Technical Paper, 2004-01-2088.

10. Nguyen M.Q., Canale M., Sename O. and Dugard L. A Model Predictive Control approach for semiactive suspension control problem of a full car, Proceedings of the IEEE $55^{\text {th }}$ Conference on Decision and Control, 2016, 721-726.

11. Hu Y., Chen M.Z.Q. and Hou Z. Multiplexed model predictive control for active vehicle suspensions, Int. J. of Ctrl., 2015, 88(2), 347-363. 
12. Mehra R.K., Amin J.N., Hedrick K.J., Osorio C. and Gopalasamy S. Active Suspension Using Preview Information and Model Predictive Control, Proceedings of the IEEE International Conference on Control Applications, 1997, 860-865.

13. Ahmed M. and Svaricek F. Preview Optimal Control of Vehicle Semi-active Suspension Based on Partitioning of Chassis Acceleration and Tire Load Spectra, Proceedings of the IEEE European Control Conference, 2014, 1669-1674.

14. De Bruyne S., Van der Auweraer H. and Anthonis J. Preview Control of a Constrained Hydraulic Active Suspension System, Proceedings of the $51^{\text {st }}$ IEEE Conference on Decision and Control, 2015, 4400-4405.

15. Savaresi S.M., Poussot-Vassal C., Spelta C., Sename O. and Dugard L. Semi-Active Suspension Control Design for Vehicles. Chapter 5: Optimal Strategy for Semi-Active Suspensions and Benchmark, Elsevier, 2010, 91-106.

16. Göhrle C., Wagner A., Schindler A. and Sawodny O. Active Suspension Controller using MPC based on a full-car model with preview information, Proceedings of the IEEE American Control Conference, 2012, 497-502.

17. Donahue M.D. and Hedrick J.K. Implementation of an Active Suspension, Preview Controller for Improved Ride Comfort, In: Nonlinear and Hybrid Systems in Automotive Control, Springer, 2003.

18. Bemporad A., Morari M., Dua V. and Pistikopoulos E.N. The Explicit Solution of Model Predictive Control via Multiparametric Quadratic Programming, Proceedings of the IEEE American Control Conference, 2000, 2, 872-876.

19. Bemporad A., Borrelli F. and Morari M. Model Predictive Control Based on Linear Programming - The Explicit Solution, IEEE Trans. Autom. Ctrl., 2002, 47(12), 1974-1985.

20. Giorgetti N., Bemporad A. and Tseng H.E. Hybrid Model Predictive Control Application Towards Optimal Semi-Active Suspension, Proceedings of the IEEE International Symposium on Industrial Electronics, 2005, 391-398.

21. Csekő L.H., Kvasnica M. and Lantos B. Analysis of the explicit model predictive control for semiactive suspension, Polytech. Elec. Eng., 2010, 54(1-2), 41-58.

22. Canale M., Milanese M., Novara C. and Ahmad Z. Semi-Active Suspension Control Using "Fast" Model Predictive Techniques, IEEE Trans. Ctrl. Syst. Tech., 2006, 14, 1034-1046.

23. Paschedag T., Giua A. and Seatzu C. Constrained optimal control: an application to semiactive suspension systems, Int. J. of Syst. Sc., 2010, 41(7), 797-811.

24. Fallah S., Sorniotti A. and Gruber P. A Novel Robust Optimal Active Control of Vehicle Suspension Systems, IFAC Proceedings, 2014, 47(3), 11213-11218.

25. Monroe Intelligent Suspension Products, http://www.monroeintelligentsuspension.com/products/.

26. Kvasnica M. Multi-Parametric Toolbox 3, http://people.ee.ethz.ch/ mpt/3/, 2014.

27. Geyer T., Torrisi F.D. and Morari M. Optimal complexity reduction of polyhedral piecewise affine systems, Automatica, 2008, 44, 1728-1740.

28. Theunissen J., Sorniotti A., Gruber P., Fallah S., Dhaens M., Reybrouck K., Lauwerys C., Vandersmissen B., Al Sakka M., Motte K. Explicit model predictive control of active suspension systems, Proceedings of the International Conference on Advanced Vehicle Powertrains, 2017, 344362. 